\title{
Global Polio Eradication: So Close, yet so Far
}

\author{
Samad EJ Golzari ${ }^{1}$, Ata Mahmoodpoor ${ }^{2 *}$, Babak Sabermarouf ${ }^{3}$
}

1. Liver and Gastrointestinal Disease Research Center, Tabriz University of Medical Sciences, Tabriz, Iran

2. Cardiovascular Research Center, Tabriz University of Medical Sciences, Tabriz, Iran

3. Neurosciences Research Center (NSRC), Tabriz University of Medical Sciences, Tabriz, Iran

Launched in 1988, the Global Polio Eradication Initiative (GPEI) never seemed as close to its sole target as by 2012; polio cases decreased by over $99 \%$ thanks to the global efforts restricting endemic areas into three countries, i.e. Afghanistan, Pakistan and Nigeria. However, the enchantment did not last long as WPV1 and confirmed new polio cases were reported in Syria (1). Reemergence of the virus, related to the WPV1 still endemic in Pakistan, had already been identified in some of the Middle Eastern countries and caused an outbreak in China (2). In previous experiences, for instance in Egypt, international outbreak response guidelines issued by the World Health Assembly (WHA) in Resolution WHA59 were implemented comprehensively and supplementary immunization activities (SIAs) were conducted carefully. Such global measures should immediately be taken in order to halt the risk of transmission and spread of WPV as a public health threat (2).

Thanks to the continuing support of WHO and Iranian health ministry, Iran is the only country which has achieved the goal of polio eradication yet neighbouring two of the three polio endemic countries. However, the on-going challenge has become even more daunting as the threat has the potential of exacerbation due to the imported cases or carriers. Although Iran has been successful in eradication of some infectious diseases, imported cases contribute immensely to the failure in realizing the eradication aim for some other infectious diseases $(3,4)$. Bordering Pakistan (909 km (565 mi)) and Afghanistan (936 km (582 mi)) to the east is one of the few major obstacles to be perceived as important. In some parts of Pakistan, immunization activities were suspended since June 2012 (2). It is pivotal to the polio eradication goals that immunization is restarted in the halted areas and reinforced in neighboring high-risk areas in order to prevent further spread of this outbreak.

Surveillance for cases of acute flaccid paralysis (AFP) should be reinforced in all countries, especially those neighboring polioinfected countries and those susceptible to contact with immigrants and frequenttravellers. Furthermore, to perform supplementary immunization activities and

\section{Corresponding author:}

Ata Mahmoodpoor, MD

Cardiovascular Research Center, Tabriz University of Medical Sciences, Tabriz, Iran

E-mail: amahmoodpoor@yahoo.com, Tel: +989141160888 
to minimize the concerns of any probable virus re-emergence routine immunization coverage data should be evaluated by all countries and global prioritization should be directed to areas at high-risk of importations and those with OPV3/DPT3 coverage of less than $80 \%$. It is of immense importance not to forget that children in all countries are at risk of contracting polio until a single child remains infected.

\section{References:}

1. World Health Organization. Global Alert and Response. Polio in the Syrian Arab Republic. http://www.who.int/csr/don/2013_10_29/en/index.html. (accessed Nov 30, 2013).

2. WHO. Polio and Prevention. http://www.who.int/entity/csr/don/archive/disease/poliomyelitis/en/index.html . (accessed Nov 30, 2013).

3. Golzari SE, Ghabili K, Bazzazi AM, Aslanabadi S. World Leprosy Day: where does Iran stand? Lancet. 26;381(9863):e3.

4. Golzari SE, Ghabili K, Khanli HM, Mahmoodpoor A, Sabermarouf B. Imported malaria cases: a source of moving backward from elimination to pre-elimination phase in malaria control. Clin Infect Dis. 2013;57(7):1061-2. 